\title{
Uniqueness and Nonuniqueness of the GNSS Carrier-Phase Compass Readings
}

\author{
P.J.G. Teunissen
}

\begin{abstract}
In this contribution we analyse the possible nonuniqueness in the least-squares solution of the GNSS carrier-phase compass model. It is shown that this lack of uniqueness may manifest itself in the fixed baseline estimator and therefore in the GNSS compass readings. We present the conditions under which nonuniqueness occurs and give explicit expressions for these nonunique least-squares solutions.
\end{abstract}

Keywords

GNSS-compass • Ambiguity resolution • Attitude nonuniqueness

\section{Introduction}

Global Navigation Satellite System (GNSS) attitude determination is a field with a wide variety of challenging (terrestrial, air and space) applications, see e.g. Cohen (1992), Lu (1995), Tu et al. (1996), Montgomery et al. (1997), Park and Teunissen (2003), Simsky et al. (2005), Kuylen et al. (2006), Teunissen (2006), Hide and Pinchin (2007).

In the present contribution we consider the determination of heading and elevation (or yaw and pitch) and therefore restrict ourselves to the two-antenna, single baseline case. GNSS carrier phase data and integer ambiguity resolution are needed in order to determine the compass parameters with the highest possible precision. Short baseline, epoch-by-epoch,

P.J.G. Teunissen $(\bowtie)$

Delft Institute of Earth Observation and Space Systems, Delft University of Technology, The Netherlands

Department of Spatial Sciences, Curtin University of Technology, Perth, Australia

e-mail: p.j.g.teunissen@tudelft.nl successful ambiguity resolution is however only possible if two or more frequencies are used, but not in the single-frequency case. In the single-frequency case, the underlying model is too weak to ensure a sufficiently high probability of correct integer estimation. Hence, for the single-frequency case, the corresponding GNSS model needs to be strengthened and this can be done by considering the length of the (small) baseline to be known. This model is referred to as the GNSS compass model and it differs from the standard GNSS single baseline model in that the known length of the baseline is added as a (weighted) constraint.

The inclusion of the baseline length constraint strengthens the model, thereby increasing the ambiguity success rates significantly, but at the same time it also complicates the least-squares estimation process. This is particularly true for short to veryshort GNSS baselines (less than $1 \mathrm{~m}$ ), as a reduction in baseline length increases the nonlinearity of the curved manifold. Related to the high-nonlinearity is another potential complication, namely the occurrence of singularities in the solution process. We will show that non-uniqueness in the attitude solutions may indeed 
occur and we will characterize them for different measurement scenarios. Singularities of different types may occur and we will identify them in relation to various data subspaces. The theoretical analysis presented also improves our understanding of the nearsingular situations. Due to a lack of space, the theorems are presented without an extensive proof. These will be published elsewhere.

\section{The GNSS Compass Model}

In principle all GNSS baseline models can be cast in the following frame of linear(ized) observation equations,

$$
\mathrm{E}(y)=A a+B b, \quad \mathrm{D}(y)=Q_{y y}, \quad a \in \mathbb{Z}^{p}, b \in \mathbb{R}^{n}
$$

where $y$ is the given GNSS data vector of order $m$, and $a$ and $b$ are the unknown parameter vectors of order $p$ and $n$ respectively. $\mathrm{E}($.$) and \mathrm{D}($.$) denote the$ expectation and dispersion operator, and $A$ and $B$ are the given design matrices that link the data vector to the unknown parameters. Matrix $A$ contains the carrier wavelengths and the geometry matrix $B$ contains the receiver-satellite unit line-of-sight vectors. The variance matrix of $y$ is given by the positive definite matrix $Q_{y y}$. The data vector $y$ will usually consist of the 'observed minus computed' single- or multi-frequency double-difference (DD) phase and/or pseudorange (code) observations accumulated over all observation epochs. The entries of vector $a$ are then the DD carrier phase ambiguities, expressed in units of cycles rather than range. They are known to be integers, $a \in \mathbb{Z}^{p}$. The entries of the vector $b$ will consist of the remaining unknown parameters, such as baseline components (coordinates) and possibly atmospheric delay parameters (troposphere, ionosphere). They are known to be real-valued, $b \in \mathbb{R}^{n}$. Vectors $a$ and $b$ are referred to as the ambiguity vector and baseline vector, respectively.

Since we consider the GNSS-Compass application in the present contribution, we restrict attention to the case of satellite tracking with two near-by antennas. The short distance between the two antennas implies that we may neglect the (differential) atmospheric delays. Thus $b$ consists then only of the three coordinates of the between baseline vector of the two antennas.

If we may assume that the two antennas are firmly attached to the body of the moving platform, the length of the baseline vector may be determined a priori. In that case we can strengthen the GNSS model (14.1) by including the additional observation equation

$$
\mathrm{E}(l)=\|b\|, \quad \mathrm{D}(l)=\sigma_{l}^{2}
$$

The required compass information (e.g. heading and pitch) follows from the baseline solution of the GNSS compass model (14.1) and (14.2). To obtain the most precise compass information, use needs to be made of the very precise carrier phase data. The inclusion of the carrier phase data into the model accounts for the presence of the unknown integer ambiguity vector $a$ in (14.1).

\section{The Least Squares Compass Solution}

The least-squares (LS) objective function of the GNSS compass model (14.1) and (14.2) is given as $H(a, b)=$ $\|y-A a-B b\|_{Q_{y y}}^{2}+\sigma_{l}^{-2}(l-\|b\|)^{2}$, with $a \in \mathbb{Z}^{p}$, $b \in \mathbb{R}^{n}$, and $\|\cdot\|_{Q_{y y}}^{2}=(.)^{T} Q_{y y}^{-1}($.$) . The LS parameter$ solution is therefore given by the minimizers

$$
\begin{aligned}
& \check{a}=\arg \min _{a \in \mathbb{Z}^{p}}\left[\min _{b \in \mathbb{R}^{n}} H(a, b)\right] \\
& \check{b}=\arg \min _{b \in \mathbb{R}^{n}} H(\check{a}, b)
\end{aligned}
$$

This can be worked out further if we let $\hat{a}$, with variance matrix $Q_{\hat{a} \hat{a}}$, denote the $\mathrm{LS}$ ambiguity solution of (14.1) without the integer constraint $a \in \mathbb{Z}^{n}$, and let $\hat{b}(a)$, with variance matrix $Q_{\hat{b}(a) \hat{b}(a)}$, denote the conditional LS baseline solution of (14.1) assuming $a$ known. Then the LS solution (14.3) can be shown to work out as

$$
\begin{aligned}
& \check{a}=\arg \min _{a \in \mathbb{Z}^{p}}\left(\|\hat{a}-a\|_{Q_{\hat{a} \hat{a}}}^{2}+\min _{b \in \mathbb{R}^{n}} G(a, b)\right) \\
& \check{b}=\arg \min _{b \in \mathbb{R}^{n}} G(\check{a}, b)
\end{aligned}
$$

where $G(a, b)=\|\hat{b}(a)-b\|_{Q_{\hat{b}(a) \hat{b}(a)}^{2}}^{2}+\sigma_{l}^{-2}(l-\|b\|)^{2}$.

Note that (14.4) reduces to the LS parameter solution of the GNSS-baseline model (14.1) in case $\sigma_{l}^{2}=\infty$. Then $\min _{b \in \mathbb{R}^{n}} G(a, b)=0$ and $\arg \min _{b \in \mathbb{R}^{n}}$ $G(a, b)=\hat{b}(a)$, from which it follows that the minimizers of $H(a, b)$ are given as

$$
\check{a}=\arg \min _{a \in \mathbb{Z}^{n}}\|\hat{a}-a\|_{Q_{\hat{a} \hat{a}}}^{2} \text { and } \check{b}=\hat{b}(\check{a})
$$


This is the commonly used solution for real-time kinematic (RTK) GNSS baseline processing, see e.g. Strang and Borre (1997), Teunissen and Kleusberg (1998), Misra and Enge (2001), Hofmann-Wellenhoff and Lichtenegger (2001), Leick (2003).

The computational complexity of (14.5) resides in the computation of the integer least-squares (ILS) solution $\check{a}$. Its computation is based on an integer search inside an ellipsoidal search space, which can be efficiently executed by means of the standard LAMBDA method, see Teunissen (1994) and Teunissen (1995). The computation of $\check{b}=\hat{b}(\check{a})$, the so-called fixed baseline, is straightforward once $\check{a}$ is known.

In our present case, we have $\sigma_{l}^{2} \neq \infty$. This increases the computational complexity considerably. First, the computation of the fixed baseline vector is more complicated; compare (14.4) with (14.5). Second, the computation of $\check{a}$ is now based on an integer search in a non-ellipsoidal search space. An efficient method for this search has been developed, see Teunissen (2006), Buist (2007), Park and Teunissen (2007), Giorgi et al. (2008).

\section{Nonuniqueness of Compass Solution}

Note that the minimization problem $\min _{b \in \mathbb{R}^{n}} G(a, b)$ of (14.4), is part of the ambiguity objective function. Thus for every evaluation of the ambiguity objective function, this minimization problem needs to be solved. A proper understanding of this minimization problem is therefore essential for the GNSS-compass ambiguity resolution problem. To simplify notation, we define

$$
F(b)=\left\|b_{0}-b\right\|_{Q}^{2}+\sigma_{l}^{-2}(l-\|b\|)^{2}
$$

Then, for $Q=Q_{\hat{b}(a) \hat{b}(a)}$, we have $\min _{b} G(a, b)=\min _{b}$ $F(b)$ if $b_{0}=\hat{b}(a)$, and $\breve{b}=\arg \min _{b} F(b)$ if $b_{0}=\hat{b}(\check{a})$, see (14.3) and (14.4). Since all the properties of the fixed baseline estimator can be derived from $F(b)$, we use from now on the simplified notation of (14.6).

The minimization of (14.6) is a nonlinear leastsquares problem of which the manifold is highly curved if $Q$ is large and $l$ is small. This is the typical case for the GNSS compass, where the baseline is very short and the single-epoch solution is determined by the relative poor code data.
The problem of minimizing $F(b)$ can be described in geometric terms as the problem of finding a point of contact between the $b_{0}$-centred ellipsoid $\| b_{0}-$ $b \|_{Q}^{2}=$ constant and the origin-centred sphere $\|b\|^{2}=$ constant. These points of contact are easily determined in case $Q$ is a scaled unit matrix, but not so in the general case.

We have the following theorem.

Theorem 1. Let $M_{b}=Q^{-1}+\sigma_{l}^{-2}(1-l /\|b\|) I_{n}$. Then $F(\hat{b}) \leq F(b)$ for all $b \in \mathbb{R}^{n}$ if and only if $M_{\hat{b}} \hat{b}=$ $Q^{-1} b_{0}$ and $M_{\hat{b}} \geq 0$.

This theorem formulates necessary and sufficiency conditions for $\hat{b}$ to be a global minimizer of $F(b)$. It also provides the conditions for having nonunique minimizers. Note that $M_{b}=M_{\hat{b}}$ for any $b \neq \hat{b}$ that has the same length as $\hat{b},\|b\|=\|\hat{b}\|$. Thus for nonunique minimizers to exist, it is necessary that $M_{\hat{b}}$ is singular.

It can be shown that $M_{b}$ is singular if and only if $\|b\|=l /\left(1+\sigma_{l}^{2} \lambda_{1}\right)$, where $\lambda_{1}$ is the smallest eigenvalue of $Q^{-1}$. Nonunique minimizers, if they exist, lie therefore all on the sphere with radius $\gamma_{1}$.

\section{When do the Nonunique Solutions Exist?}

Before we can determine the nonunique solutions, we first need to know whether they exist. The consistency requirement of the system of equations, $M_{b} b=$ $Q^{-1} b_{0}$ and $\|b\|=\gamma_{1}$, results in two conditions that the data vector $b_{0}$ has to satisfy. The first condition is that $b_{0}$ must lie in the range space of matrix $Q M_{b}, b_{0} \in$ $R\left(Q M_{b}\right)$. The second condition comes into play when the first condition is satisfied. This second condition puts restrictions on the length of $b_{0}$. Not every $b_{0}$ that makes $M_{b} b=Q^{-1} b_{0}$ consistent, will namely produce a solution that satisfies $\|b\|=\gamma_{1}$.

We start with the first condition. Let the orthogonal matrix of eigenvectors of $Q^{-1}$ be partitioned as $U=$ $\left(U_{1}, U_{2}\right)$, with $U_{1}$ containing all eigenvectors having $\lambda_{1}$ as eigenvalue. Then the null space and range space of $Q M_{b}$ are spanned by the columns of $U_{1}$ and $U_{2}$, respectively. Hence, the first consistency condition can be formulated as

$$
U_{1}^{T} b_{0}=0 \text { or } b_{0} \in R\left(U_{2}\right)
$$


If this condition is satisfied, then $M_{b} b=Q^{-1} b_{0}$ is solvable and its solutions can be expressed as

$$
b=b_{p}+U_{1} \beta_{1}
$$

where $b_{p}=\left(Q M_{b}\right)^{+} b_{0}$ is a particular solution, with $\left(Q M_{b}\right)^{+}$the pseudo inverse of $Q M_{b}$, and where $U_{1} \beta_{1}$ is the homogeneous solution, with $\beta_{1}$ still undetermined.

Since $b$ of (14.8) has to satisfy $\|b\|=\gamma_{1}$, the as yet undetermined $\beta_{1}$ cannot take on values freely, but has to satisfy

$$
\left\|\beta_{1}\right\|^{2}=\gamma_{1}^{2}-\left\|\left(Q M_{b}\right)^{+} b_{0}\right\|^{2} \geq 0
$$

This shows that $b_{0}$ may not have an arbitrary length. Hence, the nonnegativity condition of (14.9) is the second consistency condition that $b_{0}$ has to satisfy.

If we take the two conditions, (14.7) and (14.9), together, we may summarize our result as follows.

Theorem 2a. The function $F(b)$ (cf. (14.6)) has nonunique minimizers if and only if

$$
b_{0} \in \mathbb{C}=\left\{x \in \mathbb{R}^{n} \mid\|x\|_{\Sigma}^{2} \leq \gamma_{1}^{2}, x \in R\left(U_{2}\right)\right\}
$$

where $\Sigma^{-1}=U_{1} U_{1}^{T}+\left(Q M_{b}\right)^{+T}\left(Q M_{b}\right)^{+}$.

Thus we now know, if the data vector $b_{0}$ lies in the intersection of $R\left(U_{2}\right)$ and the origin-centred ellipsoidal region $\|x\|_{\Sigma}^{2} \leq \gamma_{1}^{2}$, that we will have more than one minimizer of $F(b)$.

\section{The Nonunique Solutions Determined}

It is now not difficult anymore to determine the nonunique solutions. We already know that the nonunique minimizers, if they exist, lie all on the sphere with radius $\gamma_{1}$, denoted as $S_{\gamma_{1}}$. This combined with the general solution of $M_{b} b=Q^{-1} b_{0}$ (cf. (14.8)), gives the following result.

Theorem 2b. The nonunique minimizers of $F(b)$ (cf. (14.6)), if they exist, are given by the solution set

$$
\mathbb{S}=\left\{b_{p}+R\left(U_{1}\right)\right\} \cap S_{\gamma_{1}}
$$

where $b_{p}=\left(Q M_{b}\right)^{+} b_{0}$.
The consistency set $\mathbb{C}$ and solution set $\mathbb{S}$ can both be given a clear geometric interpretation. The set $\mathbb{C}$ describes the two consistency conditions (14.7) and (14.9). Geometrically this set describes the intersection of an origin-centred ellipsoidal region with the linear manifold $R\left(U_{2}\right)$. Since $R\left(U_{1}\right)$ and $R\left(U_{2}\right)$ are each others orthogonal complement, we have $\operatorname{dim} R\left(U_{2}\right)=n-\operatorname{dim} R\left(U_{1}\right)$. Thus if $\operatorname{dim} R\left(U_{1}\right)=n$, then $\operatorname{dim} R\left(U_{2}\right)=0$ and $\mathbb{C}=\{0\}$, and if $\operatorname{dim} R\left(U_{1}\right)=$ $n-1$, then $\operatorname{dim} R\left(U_{2}\right)=1$ and $\mathbb{C}$ reduces to an origin centred interval.

The solution set $\mathbb{S}$ is the intersection of the linear manifold $b_{p}+R\left(U_{1}\right)$ with the sphere $S_{\gamma_{1}}$. It consists of two points if the linear manifold is a straight line $\left(\operatorname{dim} R\left(U_{1}\right)=1\right)$ and it forms a circle if the linear manifold is a plane $\left(\operatorname{dim} R\left(U_{1}\right)=2\right)$. Since the dimension of the linear manifold is equal to the number of times the eigenvalues of $Q^{-1}$ are equal to $\lambda_{1}$, we have $1 \leq \operatorname{dim} R\left(U_{1}\right) \leq n$. If $\operatorname{dim} R\left(U_{1}\right)=n$, then $b_{p}+R\left(U_{1}\right)=\mathbb{R}^{n}$ and $\mathbb{S}=S_{\gamma_{1}}$. This is the special case when all eigenvalues of $Q^{-1}$ are equal (i.e. $Q$ is a scaled unit matrix).

As an illustration, we now show for the cases $n=$ $1,2,3$ how the sets $\mathbb{C}$ and $\mathbb{S}$ may look like.

Case $n=1$ : If $n=1$, then $U_{1}=1, U_{2}=0$, and $Q=\lambda_{1}^{-1}$. Therefore $\mathbb{C}=\{0\}$ and $\mathbb{S}=S_{\gamma_{1}}=\{b \in \mathbb{R} \mid$ $\left.b= \pm \gamma_{1}\right\}$. Since $F(b)=\sigma_{l}^{-2}\left(1+\sigma_{l}^{2} \lambda_{1}\right)\left(b^{2}-2 \gamma_{1}|b|+\right.$ $\left.l \gamma_{1}\right)$ for $b_{0}=0$, it is readily verified that $\pm \gamma_{1}$ are indeed its two minimizers (see Fig. 14.1).

Case $n=2$ : We now have two cases: (a) $U_{1}=I_{2}$, $U_{2}=0$ and (b) $U_{1}=u_{1}, U_{2}=u_{2}$. In case (a) we have $\mathbb{C}=\{0\}$ and $\mathbb{S}=S_{\gamma_{1}}$. In case (b), $\mathbb{C}$ is an origincentred interval of length $2 \gamma_{1}\left(1-\lambda_{1} / \lambda_{2}\right)$ along the $u_{2}$ direction and $\mathbb{S}$ consists of the two intersection points of the line $b=b_{p}+u_{1} \alpha$ with the circle $S_{\gamma_{1}}$ (see Fig. 14.2). Note, if $b_{0}$ lies on the edge of $\mathbb{C}$ and thus
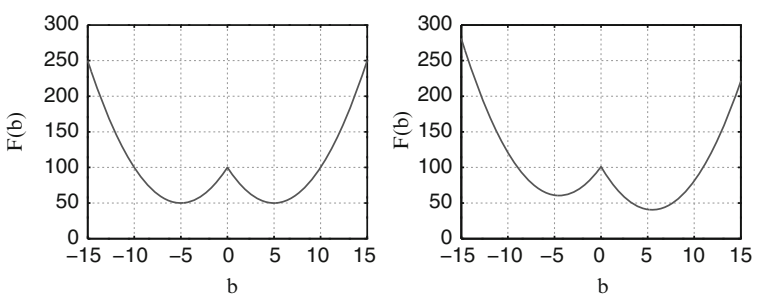

Fig. 14.1 The function $F(b)=\lambda_{1}\left(b_{0}-b\right)^{2}+\sigma_{l}^{-2}(l-|b|)^{2}$ for $b_{0}=0$ (left) and $b_{0} \neq 0$ (right) 


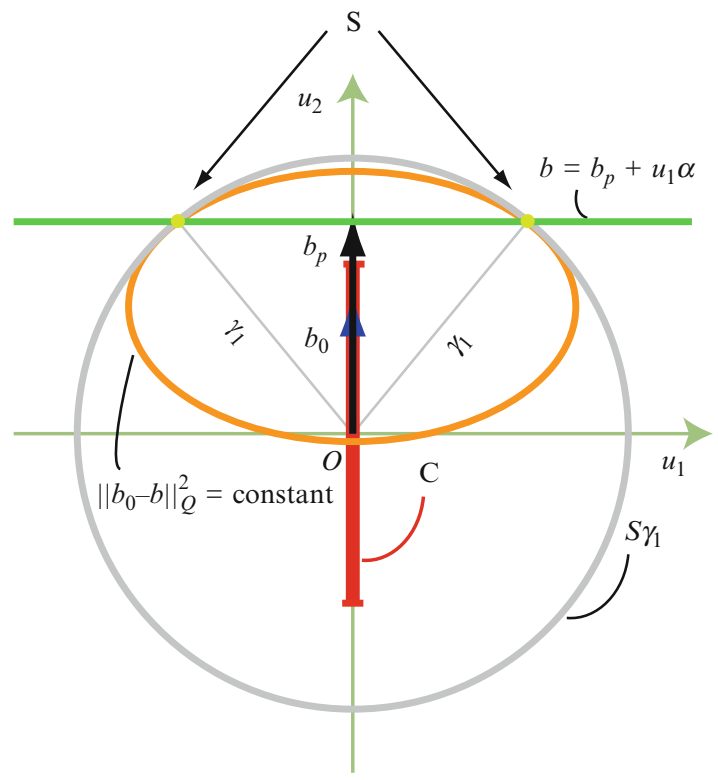

Fig. 14.2 The case $n=2$ with $U_{1}=u_{1}, U_{2}=u_{2}$ : Shown are the circle $S_{\gamma_{1}}$ with axes $u_{1}$ and $u_{2}$, the interval $\mathbb{C}$, the two solution points of $\mathbb{S}$, the line $b=b_{p}+u_{1} \alpha$ and the ellipse $\left\|b_{0}-b\right\|_{Q}^{2}=$ constant

$\left\|b_{0}\right\|=\gamma_{1}\left(1-\lambda_{1} / \lambda_{2}\right)$, that $\left\|b_{p}\right\|=\gamma_{1}$ and the two intersection points coincide in one point.

Case $n=3$ : We have the three cases: (a) $U_{1}=I_{3}$, $U_{2}=0$, (b) $U_{1}=\left(u_{1}, u_{2}\right), U_{2}=u_{3}$, and (c) $U_{1}=$ $u_{1}, U_{2}=\left(u_{2}, u_{3}\right)$. In case (a) we have $\mathbb{C}=\{0\}$ and $\mathbb{S}=S_{\gamma_{1}}$. In case (b), $\mathbb{C}$ is an origin-centred interval of length $2 \gamma_{1}\left(1-\lambda_{1} / \lambda_{3}\right)$ along the $u_{3}$ direction and the solution space $\mathbb{S}$ is the circle with centre $b_{p}$ and radius $\sqrt{\gamma_{1}^{2}-\left\|b_{p}\right\|^{2}}$ that follows from intersecting the plane $b=b_{p}+u_{1} \alpha_{1}+u_{2} \alpha_{2}$ with the sphere $S_{\gamma_{1}}$. In case (c), $\mathbb{C}$ is an origin-centred ellipse in the $U_{2}$-plane with principal axes $\gamma_{1}\left(1-\lambda_{1} / \lambda_{2}\right) u_{2}$ and $\gamma_{1}\left(1-\lambda_{1} / \lambda_{3}\right) u_{3}$. The solution space $\mathbb{S}$ is then the two point intersection of the line $b=b_{p}+u_{1} \alpha$ with the sphere $S_{\gamma_{1}}$.

The latter case $(n=3$ (c)) is the one that is most likely to occur with GNSS, since the eigenvalues of the variance matrix $Q=Q_{\hat{b}(a) \hat{b}(a)}$ will usually all be different.

Acknowledgements The research of the author has been supported by an Australian Research Council Federation Fellowship (project number FF0883188). This support is gratefully acknowledged.

\section{References}

Buist P (2007) The baseline constrained LAMBDA method for single epoch, single frequency attitude determination applications. Proceedings of ION GPS-2007, 2007

Cohen CE (1992) Attitude determination using GPS. PhD Thesis, Stanford University

Giorgi G, Teunissen PJG, Buist P (2008) A search and shrink approach for the baseline constrained LAMBDA method: experimental results. Proceedings International GPS/GNSS Symposium, Tokyo, Japan, Nov. 11-14, 2008

Hide C, Pinchin DJ (2007) Development of a low cost multiple GPS antenna attitude system. Proceedings ION GNSS 2007, 88-95, 2007

Hofmann-Wellenhoff B, H Lichtenegger (2001): Global positioning system: theory and practice, 5nd edn. Springer, Berlin

Kuylen LV, Nemry P, Boon F, Simsky A, Lorga JFM (2006) Comparison of attitude performance for multi-antenna receivers. Eur J Navigation, 4(2)

Leick A (2003) GPS satellite surveying, 3rd edn. Wiley, New York

Lu G (1995) Development of a GPS multi-antenna system for attitude determination. UCGE Reports 20073, Dept. of Geomatics Eng., University of Calgary, 1995

Misra P, Enge P (2001) Global positioning system: signals, measurements, and performance. Ganga-Jamuna Press, Lincoln, MA

Montgomery P, IY Bar-Itzhack, J Garrick (1997): Algorithm for attitude determination using GPS. Proceedings of AIAA Guidance, Navigation and Control Conf., New Orleans, LA, USA. Aug. 1997, Paper no. AIAA 97-3616, 1997.

Park C, Teunissen PJG (2003) A new carrier phase ambiguity estimation for gnss attitude determination systems. Proceedings of International GPS/GNSS Symposium, Tokyo, 2003.

Park C, Teunissen PJG (2007) A baseline constrained lambda method for integer ambiguity resolution of GNSS attitude determination systems. J Control, Robotics Systems 14(6):587-594

Simsky A, Vander Kuylen L, Boon F (2005) Single-board attitude determination system based on the PolaRx2@GPS receiver. Proc ENC GNSS 2005, Munich, Germany, 19-22 July, 2005.

Strang G, Borre K (1997) Linear algebra, geodesy, and GPS. Wellesley-Cambridge Press, Wellesley, MA

Teunissen PJG (1994) A new method for fast carrier phase ambiguity estimation. Proceedings IEEE PLANS'94, Position Location and Navigation Symposium, pp 562-573, 1994

Teunissen PJG (1995) The least-squares ambiguity decorrelation adjustment: a method for fast GPS integer ambiguity estimation. J Geodesy 70:65-82

Teunissen PJG (2006) The LAMBDA method for the GNSS compass. Artificial Satellites, 41(3):89-103

Teunissen PJG, Kleusberg (eds) (1998) GPS for Geodesy, 2nd enlarged (edn) Springer, Berlin

Tu CH, KY Wang, WC Melton (1996) GPS compass: a novel navigation equipment. Proceedings of ION National Technical Meeting, Santa Monica, CA, USA, 1996 